\title{
Mast cell-derived angiopoietin-1 plays a critical role in the growth of plasma cell tumors
}

\author{
Takayuki Nakayama, Lei Yao, and Giovanna Tosato
}

Experimental Transplantation and Immunology Branch, Center for Cancer Research, National Cancer Institute, Bethesda, Maryland, USA.

\begin{abstract}
Multiple myeloma in humans is frequently associated with mast cell infiltration and neovascularization, which correlate directly with disease severity, but the mechanisms underlying this relationship remain unclear. Here, we report that primary murine mast cells express angiopoietin-1 (Ang-1) and low levels of VEGF-A but not Ang-2 and that 2 established murine plasmacytoma cell lines express high levels of VEGF-A but little or no Ang-1 or Ang-2. An in vivo angiogenesis assay using extracellular matrix components shows that mast cells and plasmacytoma cells, together, promote marked neovascularization composed of dilated vessels, which is prevented by neutralization of VEGF-A and Ang-1 but is only partially reduced by neutralization of either VEGF-A or Ang-1. Mast cells within extracellular matrix components express Ang-1, and recombinant Ang-1 together with plasmacytoma cells promotes extracellular matrix neovascularization similar to that induced by mast cells. A transplantation assay shows that primary mast cells accelerate tumor growth by established plasmacytoma cell lines and that neutralization of Ang-1 alone or with VEGF-A reduces significantly the growth of plasmacytomas containing mast cells. These results demonstrate that mast cell-derived Ang-1 promotes the growth of plasmacytomas by stimulating neovascularization and provide further evidence supporting a causal relationship between inflammation and tumor growth.
\end{abstract}

\section{Introduction}

Considerable evidence supports an initiating role of chronic inflammation in a proportion of malignancies $(1,2)$. Plasmacytomas are reproducibly induced in genetically susceptible strains of mice by i.p. injections of paraffin oils and other agents that cause chronic peritoneal inflammation, and administration of the anti-inflammatory drug indomethacin inhibits plasma cell tumor development $(3,4)$. In humans, inflammatory bowel diseases predispose to colorectal cancer (5), chronic Helicobacter pylori infection is the leading cause of gastric cancer (6), and HBV or HCV infection predisposes to liver carcinoma (7). Long-term use of NSAIDs reduces colon cancer risk by $40-50 \%$, presumably by inactivating cyclooxygenases (8). Once established, many malignancies maintain an inflammatory component, perhaps due to persistence of the inflammation-initiating factors or to recruitment of inflammatory cells from the bloodstream, resulting in various inflammatory cytokines being present at the tumor site (2).

Mast cells are commonly recognized at the margins of diverse tumors in humans and rodents (9-11). Genetic and functional experiments suggested that mast cells can promote tumorigenesis and tumor growth (12-14). In a murine model of squamous epithelial carcinogenesis, mast cells in conjunction with neutrophils and other inflammatory cells contributed to tumor growth, in part due to production of the protease MMP-9, which releases proangiogenic factors from the $\operatorname{ECM}(14,15)$. However, MMPs may serve as both promoters and inhibitors of tumor growth (16). In addition, mast cells can release numerous factors stored

Nonstandard abbreviations used: Ang, angiopoietin; BMMC, bone marrow mast cell; PIGF, placental growth factor; SPMC, spleen mast cell.

Conflict of interest: The authors have declared that no conflict of interest exists.

Citation for this article: J. Clin. Invest. 114:1317-1325 (2004).

doi:10.1172/JCI200422089. or newly synthesized after activation, including heparin, heparinase histamine, adenosine receptors, bFGF, and VEGF-A, which are known to modulate angiogenesis $(11,14,17)$.

Multiple myeloma, a malignancy characterized by the infiltration of malignant plasma cells in the bone marrow, is frequently associated with neovascularization and mast cell infiltration (18-20). Recently, vascular density and degree of mast cell infiltration in myeloma tissues were found to directly correlate with each other and with disease activity and grade $(20,21)$. This suggested a role for mast cell-induced angiogenesis in promoting growth of multiple myeloma. In this study, we have surveyed mast cells for production of proangiogenic factors, and tested whether mast cells can promote angiogenesis and plasmacytoma cell growth. We conclude that mast cell-derived angiopoietin-1 (Ang-1) plays a critical role in promoting the growth of plasma cell tumors, acting as a proangiogenic factor in conjunction with the plasmacytoma-derived VEGF-A.

\section{Results}

Expression of proangiogenic factors and their receptors in mast cells and plasmacytoma cells. Using RT-PCR, we examined mast cell and plasmacytoma cell expression of VEGF-A, VEGF-B, VEGF-C, VEGF-D, placental growth factor (PlGF), Ang-1, and Ang-2, molecules known to regulate endothelial cell function and angiogenesis $(22,23)$. All primary mast cells were derived from the bone marrow and cultured with IL-3 (referred to here as bone marrow mast cells [BMMCs]), derived from the bone marrow and cultured with a combination of SCF, IL-6, and IL-10 (referred to as TRIAD mast cells), or derived from the spleen and cultured with IL-3 (referred to as spleen mast cells [SPMCs]), and the plasmacytoma cell lines TEPC1165SZ and TEPC2027 expressed VEGF-A and VEGF-B mRNA (Figure 1A). We identified VEGF-C, PlGF, and Ang-1 expression in all mast cells but not in plasmacytoma cells. VEGF-D was 


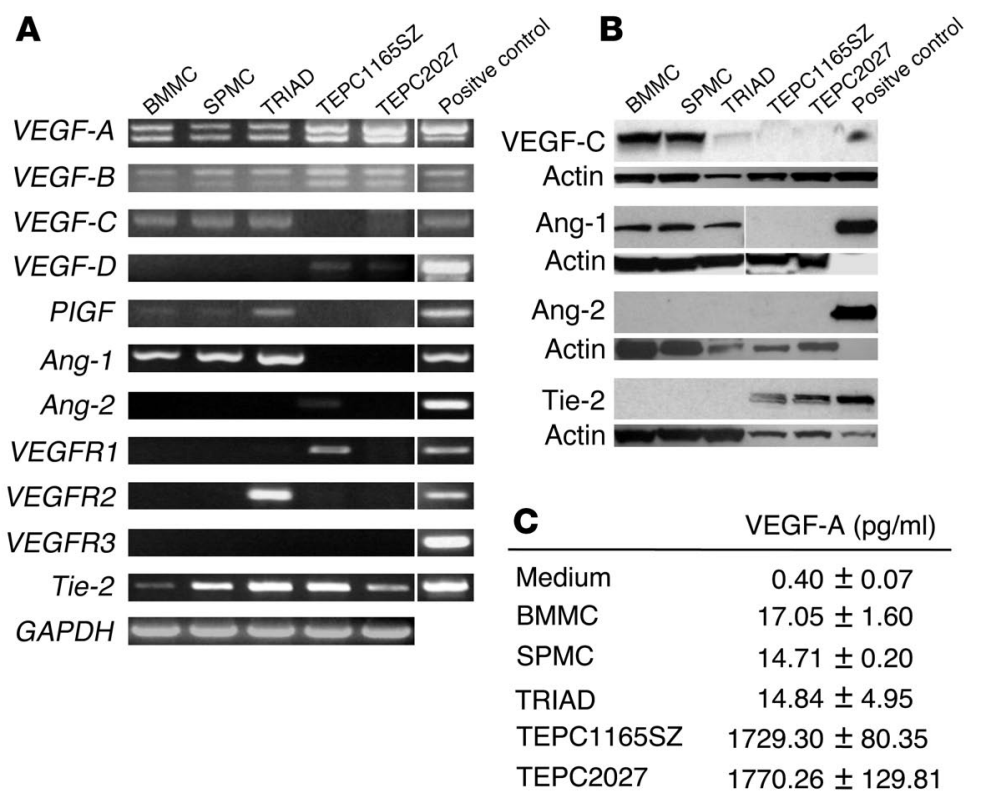

\section{Figure 1}

Expression of proangiogenic factors and receptors in mast cells and plasmacytoma cells. (A) Total RNA extracted from primary mast cells from bone marrow (BMMCs, cultured with IL-3; TRIAD cells, cultured with SCF, IL-6, and IL-10) and spleen (SPMCs, cultured with IL-3), and from plasmacytoma cell lines (TEPC1165SZ and TEPC2027), was subjected to RT-PCR using specific primer. Representative results are shown. (B) Western blot analysis of lysates from $2 \times 10^{6}$ mast cells and plasmacytoma cells using specific antibodies. Loading accuracy was evaluated by reprobing with anti-actin antibodies. Positive controls: VEGF-C, lung tissue; Ang-1, recombinant Ang-1 (2.5 ng); Ang-2, recombinant Ang-2 (2.5 ng); Tie-2, human umbilical vein EC. (C) VEGF-A content in culture supernatants of mast cells and plasmacytoma cells, detected by specific ELISA. All cells were cultured at $10^{6}$ cells/ml for 48 hours. expressed in TEPC1165SZ and TEPC2027, and Ang-2 was detected only in TEPC1165SZ. Ang-2 expression was not detectable in mast cells. To evaluate the possibility that angiogenic factors released from mast cells may act on plasmacytoma cells, we examined cell expression of receptors for VEGF and Ang-1/Ang-2, including VEGFR1, VEGFR2, VEGFR3, and the endothelial receptor tyrosine kinase Tie-2. TEPC1165SZ expressed VEGFR1 and Tie-2, whereas TEPC2027 expressed only Tie-2. All primary mast cells expressed Tie-2, and TRIAD mast cells also expressed VEGFR2.

Using cell lysates in immunoblotting experiments, we confirmed that primary mast cells (BMMCs, TRIAD cells, and SPMCs) express VEGF-C and Ang-1 proteins, but not Ang-2 (Figure 1B). The expression of Ang-1 in primary mast cells was confirmed using 3 distinct antibodies (N-18, H-98, and C-19; Santa Cruz Biotechnology Inc.) which specifically detected a band attributable to Ang-1 (results shown reflect N-18 addition; others not shown). The expression of Tie- 2 in mast cells, which was detectable by RT-PCR, was not confirmed by immunoblotting; this likely reflects low levels of Tie-2 expression in mast cells. In addition, we confirmed by immunoblotting that cell lysates from TEPC1165SZ and TEPC2027 plasmacytoma cells express Tie-2, but not Ang-1 or Ang-2 (Figure 1B).

We measured VEGF-A secretion by primary mast cells and compared it with that by plasmacytoma cells (Figure 1C). VEGF-A was detected at concentrations of $15-17 \mathrm{pg} / \mathrm{ml}$ in the culture supernatants of all primary mast cells (BMMCs, TRIAD cells, and SPMCs), but at much higher concentrations $(1.7-1.8 \mathrm{ng} / \mathrm{ml})$ in culture supernatants of TEPC1165SZ and TEPC2027 plasmacytoma cells.

Since mast cells express Ang-1 and plasmacytoma cells express the Ang-1 receptor Tie-2, we tested whether plasmacytoma cells are responsive to Ang-1. Using TEPC1165SZ and TEPC2027 plasmacytoma cells, we measured proliferation, survival, and VEGF-A secretion in the presence or absence of recombinant Ang-1 (not shown). Even at the highest concentration tested $(250 \mathrm{ng} /$ $\mathrm{ml}$ ), Ang-1 had minimal effect on the proliferation and viability of TEPC1165SZ and TEPC2027 cells ( $P>0.2$ for all determinations). Also, Ang-1 affected VEGF-A secretion by the plasmacytoma cell lines only minimally $(P>0.1)$.
Effects of mast cells on angiogenesis in vivo. Previous studies have shown that Ang-1 promotes angiogenesis on its own, and that it is additive to VEGF-A in promoting angiogenesis (24). Since we had determined that plasmacytoma TEPC1165SZ and TEPC2027 cell lines secrete nanogram-per-milliliter amounts of VEGF-A in culture, we tested for the possibility that mast cells may indirectly promote plasmacytoma cell growth by promoting angiogenesis. In an in vivo angiogenesis assay using Matrigel (BD Biosciences - Discovery Labware) mast cells (BMMCs, $0.5 \times 10^{6}$ ) and plasmacytoma cells (TEPC2027, $0.5 \times 10^{6}$ ) individually induced formation of only a few thin capillaries identified by their content of red blood cells (Figure 2A). By contrast, mast cells (BMMCs, $0.5 \times 10^{6}$ ) and plasmacytoma cells (TEPC2027, $0.5 \times 10^{6}$ ), together, induced the formation of numerous, markedly dilated vascular structures identified with Masson's trichrome staining by their content of red cells and the presence of a flattened cellular lining morphologically attributable to typical endothelium (Figure 2A). This proangiogenic effect was not noted when the plasmacytoma cells were injected alone at a higher concentration $\left(1.0 \times 10^{6}\right.$ TEPC2027 cells; not shown). Measurement of vascular structures (identified as areas within the Matrigel lined by endothelium and containing red cells) revealed that mast cells in conjunction with plasmacytoma cells were significantly $(P<0.05)$ more angiogenic compared with each cell type alone (Figure 2B, top). In addition, Matrigel plugs containing mast cells plus plasmacytoma cells appear to be substantially more cellular than plugs inoculated with each cell type alone. Using immunohistochemical staining for mouse $\kappa$ light chain (TEPC2027 cells secrete $\kappa$ chains), numerous plasmacytoma cells, often in clumps, were identified within plugs containing mast cells plus plasmacytoma cells, but fewer, scattered positive cells were identified in plugs containing plasmacytoma cells alone (Figure 2A). Quantitative analysis confirmed the presence of a significantly $(P<0.05)$ greater number of plasmacytoma cells in the plugs containing mast cells plus plasmacytoma cells compared with plugs with plasmacytoma cells alone (Figure 2B, bottom). Comparable results were derived with SPMCs (not shown), and with BMMCs in conjunction with TEPC1165SZ plasmacytoma cells (not shown). 

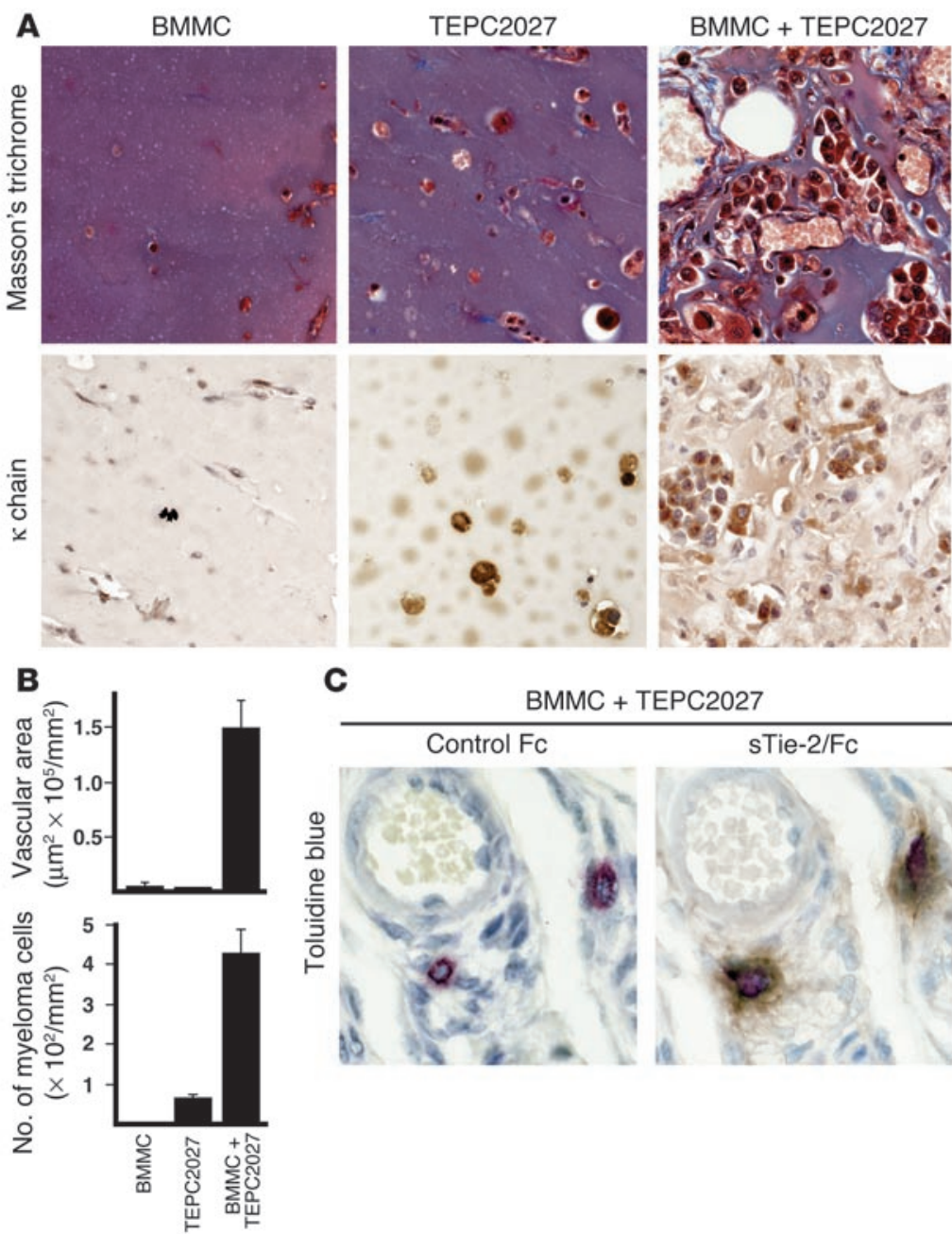

c

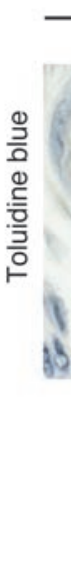

\section{Figure 2}

Effects of mast cells and plasmacytoma cells on angiogenesis in vivo. BALB/c nu/nu mice (3-5 mice per group) were inoculated s.c. with Matrigel $(0.5 \mathrm{ml})$ in conjunction with BMMCs $\left(0.5 \times 10^{6}\right)$, TEPC2027 plasmacytoma cells $\left(0.5 \times 10^{6}\right)$, or BMMCs plus TEPC2027 cells $\left(0.5 \times 10^{6}\right.$ each). Matrigel plugs, removed after 7 days, were processed for histology. (A) Top row: Representative histological images of Matrigel plugs stained with Masson's trichrome. Bottom row: Representative histological images of Matrigel plugs immunostained for $\kappa$ light chain. Original magnification, $\times 20$. (B) Quantitative analysis of neovascularization (top) and plasmacytoma cell infiltration (bottom) in all Matrigel plugs (3-5 plugs per group; 1 plug per animal). Neovascularization was measured as the areas occupied by vascular structures and is expressed as the mean surface area $( \pm S D$ of measurements from individual plugs in each group) occupied by vascular structures in each group. Plasmacytoma cell infiltration was measured by counting plasmacytoma cells present in each plug section and is expressed as the mean cell number $( \pm$ SD of measurements in individual plugs from each group). (C) Consecutive sections containing BMMCs plus TEPC2027 cells were doublestained with toluidine blue and Tie-2/Fc or control Fc. Mast cells, purple due to toluidine blue and brown due to Tie-2/Fc (but not control Fc), localize in the proximity of a vessel. Original magnification, $\times 40$.
Mast cells, identified within the plugs by cytochemical staining with toluidine blue (purple), localized mostly in the proximity of newly formed vessels (Figure 2C). Importantly, toluidine-positive cells expressed Ang-1, as they specifically stained with Tie-2/Fc (brown) but not control Fc (Figure 2C). These results demonstrate that, in the presence of plasmacytoma cells, mast cells can promote Matrigel neovascularization and growth or survival of plasmacytoma cells in vivo.

Contribution of Ang-1 to angiogenesis induced by mast cells. To test for the possibility that Ang-1 contributes to increased angiogenesis induced by mast cells, we used recombinant Tie- $2 / \mathrm{Fc}$ fusion protein to neutralize Ang-1 with or without neutralizing antibodies to VEGF-A (Figure 3). As observed above, Matrigel plugs containing both BMMCs and TEPC2027 alone (no additives) contained numerous vascular structures and clusters of $\kappa$-staining plasmacytoma cells (Figure 3, A and B). Individually, neutralizing antibodies directed at VEGF-A and Tie-2/ Fc partially reduced the area occupied by vascular structures (Figure $3 \mathrm{~B}$, top; $P=0.15$, anti-VEGF-A antibody; $P=0.02$, Tie- $2 / \mathrm{Fc}$ ) and the number of plasmacytoma cells within the plugs (Figure 3B, bottom; $P<0.05$, both conditions). Together, Tie-2/Fc- and VEGF-A-neutralizing antibodies reduced more significantly the area occupied by vascular structures $(P<0.001)$ and the number of plasmacytoma cells in the plugs $(P<0.001)$. Both parameters were only minimally affected $(P>0.1)$ by control reagents $(\mathrm{B} 7-1 / \mathrm{Fc}$ in conjunction with a control antibody; Figure 3B); this provided evidence for a proangiogenic role of mast cell-derived Ang-1. To examine this further, we used recombinant Ang-1. As shown in Figure 3, C and D, Matrigel plugs containing recombinant Ang-1 together with plasmacytoma cells displayed evidence of increased neovascularization and plasmacytoma cell infiltration compared with Matrigel plugs inoculated with plasmacytoma cells alone $(P<0.05$, both comparisons). Indeed, the dilated vascular structures noted with Ang-1 plus plasmacytoma cells resembled morphologically those derived from inoculation of mast cells with plasmacytoma cells (Figure 3A). Monocytes and macrophages were only rarely identified by cytochemical esterase staining within Matrigel plugs with or without Ang-1 (not shown). These results provide evidence that Ang-1 produced by mast cells critically contributes to increased Matrigel neovascularization in vivo.

Mast cells and Ang-1 promote plasmacytoma cell growth in vivo. We tested for the potential contribution of mast cells to plasmacytoma cell tumorigenesis in vivo (Figure 4A). TEPC1165SZ cells $\left(5 \times 10^{6}\right)$ gave rise to tumors significantly more rapidly in nu/nu BALB/c mice when they were inoculated s.c. together with mast cells (BMMCs or SPMCs, $5 \times 10^{6}$ ). Whereas tumors emerged 3-5 days after injection of TEPC1165SZ with either type of mast cells, tumors emerged only after 11-14 days after injection of TEPC1165SZ alone (Figure 4B). In contrast to the tumor-promoting activity of mast cells, no acceleration 


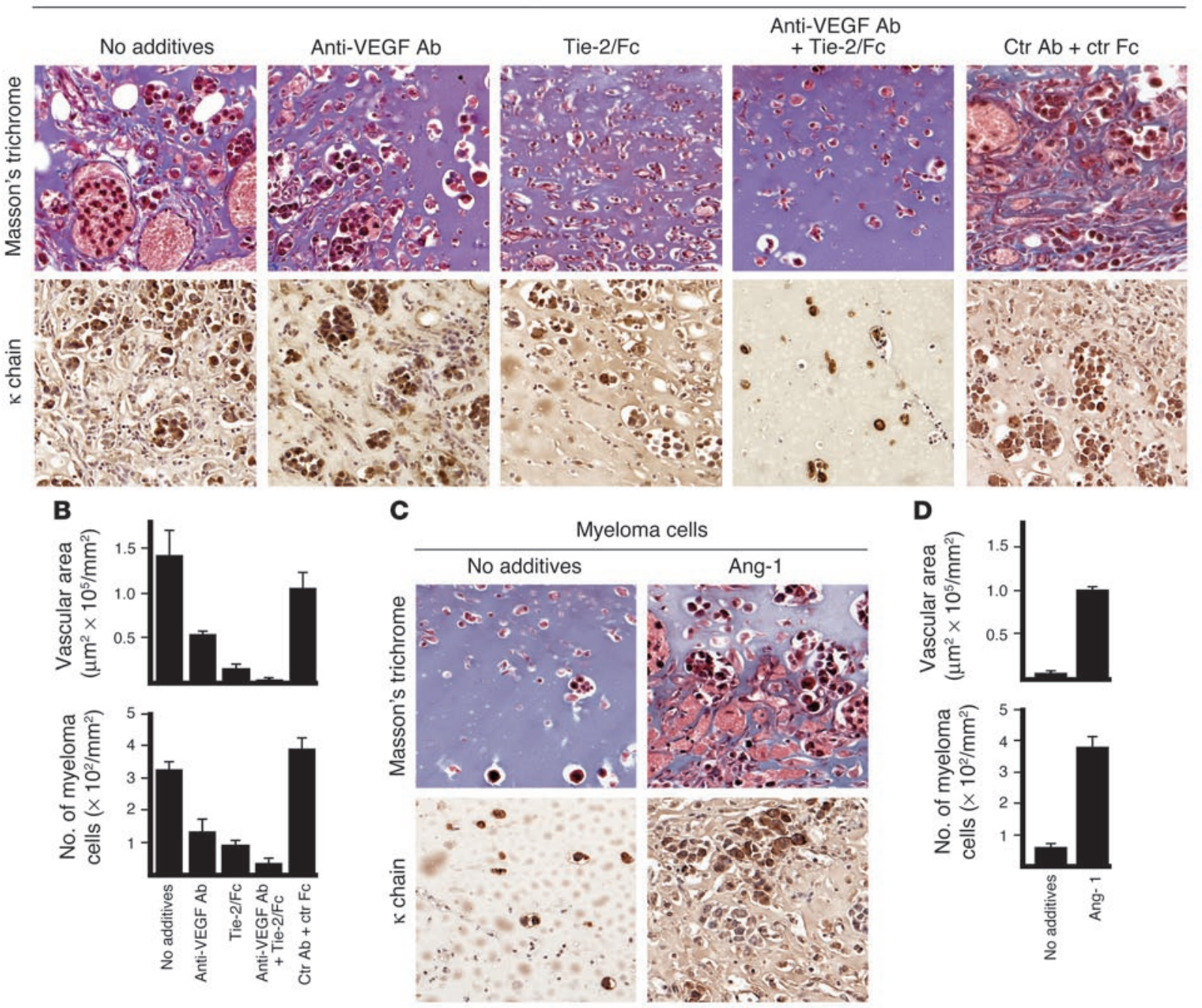

Figure 3

Evidence supporting a contribution of mast cell-derived Ang- 1 to neovascularization in vivo. Matrigel plugs ( $0.5 \mathrm{ml})$ containing plasmacytoma cells (TEPC2027 cells, $\left.0.5 \times 10^{6}\right)$ alone or with BMMCs $\left(0.5 \times 10^{6}\right)$ were inoculated s.c. into mice (3-5 mice per group) without additives or with antiVEGF-A antibody $(5 \mu \mathrm{g} / \mathrm{ml})$, Tie-2/Fc ( $5 \mu \mathrm{g} / \mathrm{ml})$, anti-VEGF-A antibody plus Tie-2/Fc (5 $\mu \mathrm{g} / \mathrm{ml}$ each), control (Ctr) goat lgG plus control B7-1/Fc (5 $\mu \mathrm{g} / \mathrm{ml}$ each), or recombinant Ang-1 (500 ng/ml). (A) Representative histological images of Matrigel plugs stained with Masson's trichrome (top row), showing different degrees of neovascularization; and immunostained for $\kappa$ light chain (bottom row), showing differing degrees of plasmacytoma cell infiltration under the experimental conditions tested. Original magnification, $\times 20$. (B) Quantitative analysis (as described in the legend to Figure 2) of Matrigel neovascularization (top) and plasmacytoma cell infiltration (bottom) under the experimental conditions tested (3-5 plugs per group; 1 plug per mouse). (C) Representative microscopic images of Matrigel plugs containing plasmacytoma cells alone or with recombinant Ang-1, stained with Masson's trichrome (top row) or immunostained for $\kappa$ chain (bottom row), showing differing degrees of neovascularization and plasmacytoma cell infiltration. (D) Quantitative analysis (as described in the legend to Figure 2) of Matrigel neovascularization (top) and plasmacytoma cell infiltration (bottom) in the presence of plasmacytoma cells alone or with recombinant Ang-1 (3-5 plugs per group; 1 plug per mouse).

of tumor development was noted when $5 \times 10^{6} \mathrm{TEPC} 1165 \mathrm{SZ}$ cells were inoculated with $5 \times 10^{6}$ freshly isolated bone marrow cells (mean tumor size and weight of tumors on day 18 were not significantly different from those of control tumors derived from inoculation of TEPC1165SZ alone). As expected, neither BMMCs nor SPMCs formed tumors (Figure 4B). All mice were sacrificed 18 days after cell inoculation. The mean size (Figure $4 \mathrm{~B})$ and weight (Figure 4C) of tumors derived from inoculation of either TEPC1165SZ plus BMMCs $\left(365.0 \mathrm{~mm}^{2}\right.$ and 2.87 g) or TEPC1165SZ plus SPMCs $\left(413.5 \mathrm{~mm}^{2}\right.$ and $\left.3.25 \mathrm{~g}\right)$ were significantly greater $(P<0.05$, all comparisons $)$ than the size and weight of tumors derived from inoculation of TEPC1165SZ alone $\left(133.0 \mathrm{~mm}^{2}\right.$ and $\left.0.80 \mathrm{~g}\right)$. In parallel experiments, none of $4 \mathrm{nu} / \mathrm{nu} \mathrm{BALB} / \mathrm{c}$ mice inoculated s.c. with $5 \times 10^{6} \mathrm{TEPC} 2027$ plasmacytoma cells formed tumors over 8 weeks' observation, but 4 of 4 developed tumors by day 28 (tumors emerged between days 21 and 28) when the TEPC2027 plasmacytoma cells $\left(5 \times 10^{6}\right)$ were inoculated s.c. together with BMMCs $\left(5 \times 10^{6}\right)$. When inoculated with $10 \times 10^{6}$ TEPC2027 plasmacytoma cells, 3 of 4 mice developed tumors over 8 weeks.

Microscopically, tumors derived from inoculation of TEPC1165SZ plasmacytoma cells together with mast cells were 
A

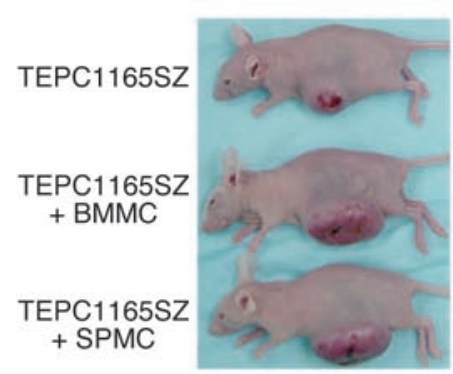

B

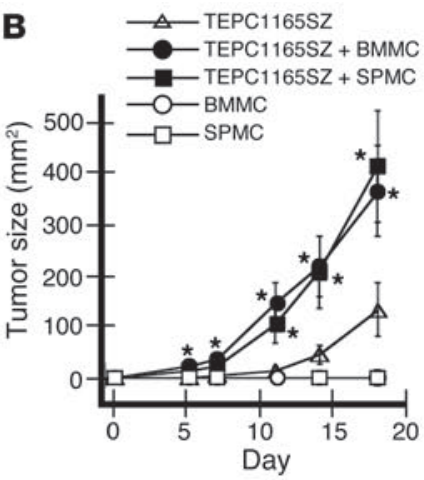

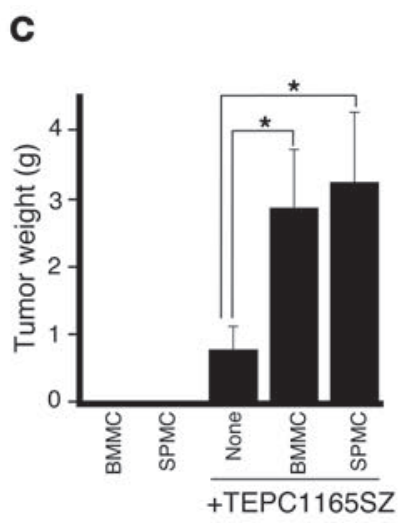

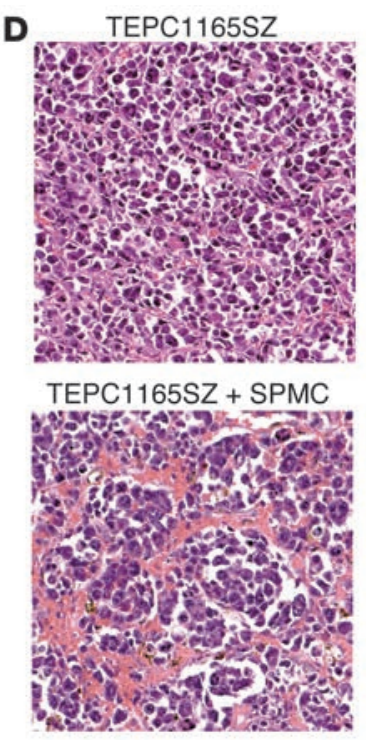

\section{Figure 4}

Effects of mast cells on plasmacytoma cell tumorigenesis in vivo. TEPC1165SZ plasmacytoma cells $\left(5 \times 10^{6}\right)$ were inoculated s.c. into BALB/c nu/nu mice either alone or in conjunction with BMMCs or SPMCs $\left(5 \times 10^{6}\right)$. There were 6 mice per group. (A) Representative images of tumorbearing mice on day 18 after initial injection. (B) Time course of tumor development. Tumor size was estimated as the product of 2-dimensional caliper measurements. The values reflect the mean tumor size expressed in square millimeters $( \pm$ SD) in each group (6 mice per group). The asterisk denotes that the mean tumor size is significantly different from that of mice inoculated with plasmacytoma cells alone. (C) Average tumor weight in grams $( \pm S D)$ measured on day 18 after cell inoculation in each group (6 mice per group). The asterisk denotes that the mean tumor weight is significantly different from that of mice inoculated with plasmacytoma cells alone. (D) Representative microscopic histology of tumor tissues from mice inoculated with TEPC1165SZ plasmacytoma cells alone or in conjunction with SPMCs. H\&E stain; original magnification, $\times 10$.

highly vascularized, whereas tumors derived from inoculation of TEPC1165SZ cells alone were generally not (Figure 4D). Cytochemical staining with toluidine blue failed to identify mast cells within tumors derived from inoculation of plasmacytoma cells alone (Figure 5A). However, rare toluidine-positive cells were identified in the dermis overlaying the tumor (not shown). By contrast, scattered mast cells were visualized both within and at the periphery of tumors derived from inoculation of plasmacytoma cells together with mast cells (Figure 5A). Ang-1 immunocytochemical staining was negative in tumors derived from inoculation of plasmacytoma cells alone (Figure 5A). Instead, scattered Ang-1-positive cells were visualized within tumors derived from inoculation of plasmacytoma cells together with mast cells (Figure 5A). Double staining showing cellular colocalization of toluidine blue and Ang-1 provided evidence that mast cells within tumors are the source of Ang-1 (Figure $5 \mathrm{~A}$, inset). In contrast to mast cells, monocytes and macrophages were rarely identified by cytochemical esterase staining within plasmacytoma tissues derived from inoculation of plasmacytoma cells alone or in conjunction with mast cells (not shown).

We tested for the contribution of Ang- 1 to mast cell-induced plasmacytoma tumor growth in vivo by using Tie-2/Fc fusion protein to neutralize Ang-1 (Figure 5B). To this end, groups of mice (4 per group) were injected s.c. with TEPC1165SZ cells in conjunction with BMMCs alone $\left(3 \times 10^{6}\right)$, with Tie- $2 / \mathrm{Fc}(50 \mu \mathrm{g}$ per mouse), with antibodies to VEGF-A (50 $\mu$ g per mouse), with Tie-2/Fc plus antibodies to VEGF-A (50 $\mu$ g each per mouse), or with control reagents (IgG/Fc plus control antibody). Additional mice (4 per group) were inoculated with TEPC1165SZ cells alone $\left(3 \times 10^{6}\right)$. As observed above, mast cells promoted significant $(P<0.05)$ plasmacytoma tumor growth in the mice (tumors derived from injection of plasmacytoma cells alone were $40.2 \%$ the size of tumors derived from injection of plasmacytoma cells plus mast cells on day 14 after injection). Neutralizing antibodies to VEGF$\mathrm{A}$ and Tie-2/Fc individually and together reduced significantly ( $P<0.05$, all determinations, day 14 after cell injection) tumor growth induced by the plasmacytoma cells in conjunction with mast cells, whereas the control reagents had minimal effect on tumor growth (Figure 5B). These results confirm that mast cells are effective at promoting the growth of plasmacytoma in vivo, and they provide evidence that Ang-1 is a critical mediator of the tumor-promoting effect of mast cells.

\section{Discussion}

Here we show that mast cells have an ability to promote the growth of plasma cell tumors through secretion of Ang-1, which stimulates neovascularization acting in conjunction with tumorderived VEGF-A. Studies in mast cell-deficient mice raised the possibility that mast cells can favor tumor growth (12), and an experimental tumor model of human papilloma virus 16 -associated squamous epithelial carcinogenesis provided evidence that inflammatory infiltrates containing mast cells and other inflammatory cells contribute to neoplastic cell proliferation and angiogenesis, in part because of MMP-9 secretion $(14,15)$. However, a unique contribution of mast cells to tumor growth has not been previously demonstrated. In addition, mast cells are known to be a source of cytokines and growth factors (17, $25,26)$, but not a source of Ang-1. Here we show that 3 distinct types of primary mast cells express Ang-1.

Angiopoietins represent a family of secreted proteins that have been linked to regulation of vasculogenesis and angiogenesis (23). 
A TEPC1165Sz

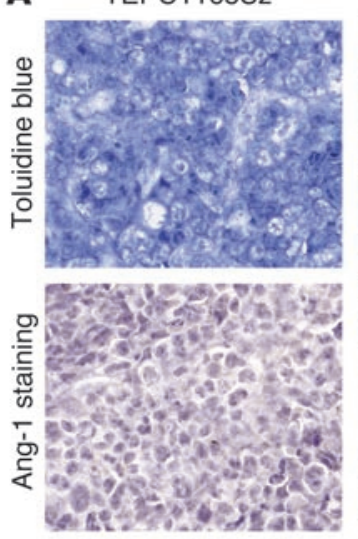

TEPC1165SZ + SPMC

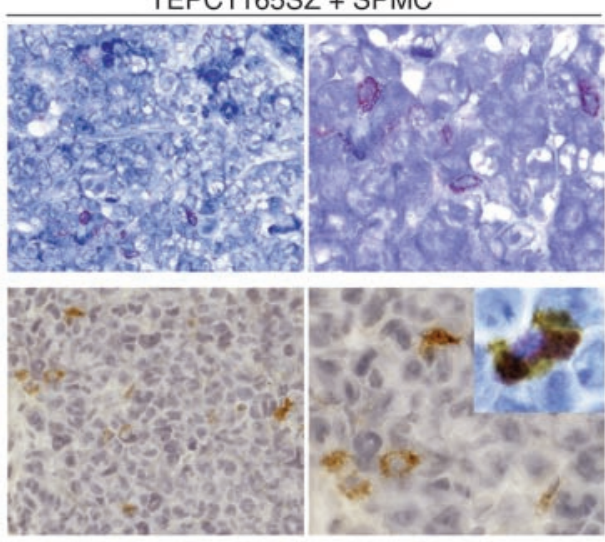

B

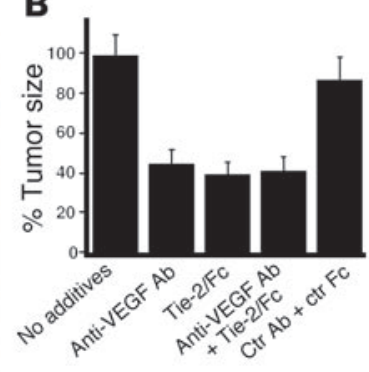

Figure 5

Contribution of Ang-1-secreting mast cells to plasmacytoma tumor growth. (A) Tumor tissues were stained with toluidine blue for detection of mast cells and/or immunostained with Tie-2/Fc for detection of Ang-1. Top row: Representative tumor tissue from a mouse inoculated with TEPC1165SZ plasmacytoma cells alone, showing no toluidine-positive cells (original magnification, $\times 20$ ), and from a mouse inoculated with TEPC1165SZ cells plus SPMCs, showing infiltration with toluidine-positive cells (original magnifications, $\times 20$ and $\times 40$ ). Bottom row: Immunostaining for Ang-1 fails to detect positive cells in representative tumor tissue from a mouse inoculated with TEPC1165SZ cells alone, but detects scattered Ang-1-positive (brown) cells in a representative tumor tissue from a mouse inoculated with SPMCs (original magnification, $\times 20$ and $\times 40$ ). Inset: Cell colocalization of toluidine blue staining and Ang-1 immunostaining (original magnification, $\times 63$ ). (B) Tumor size in mice injected s.c. with TEPC1165SZ plus BMMCs alone, Tie-2/Fc (50 $\mu \mathrm{g}$ per mouse), goat anti-mouse VEGF-A antibodies (50 $\mu \mathrm{g}$ per mouse), Tie2/Fc plus goat anti-mouse VEGF-A antibodies ( $50 \mu \mathrm{g}$ each per mouse), or human IgG Fc plus control goat IgG ( $50 \mu \mathrm{g}$ each per mouse). There were 4 mice per group. Tumor size was estimated in square millimeters on day 14 after injection; the results are expressed as the mean percent tumor size $( \pm \mathrm{SD})$ of tumors derived from inoculation of TEPC1165SZ plus BMMCs.

Ang-1 binds to and activates the receptor tyrosine kinase Tie-2, which is selectively expressed on endothelial cells (27-29), and induces sprouting and survival of endothelial cells (27). It also stimulates mobilization of vasculogenic stem cells (30). Consistently with its proangiogenic functions, Ang-1 promotes tumor angiogenesis and tumor growth in many, but not all, systems (23, $28,31)$. Ang- 1 or Tie- 2 knockout mice are embryonically lethal and display abnormalities in remodeling of the primitive vasculature $(32,33)$. Transgenic mice overexpressing Ang-1 in the skin display increased dermal vascularization (24). Double-transgenic mice overexpressing VEGF-A and Ang-1 in the skin display both enlarged microvessels attributable to Ang-1 and numerous capillary-like vessels attributable to VEGF-A, providing evidence for an additive proangiogenic effect of the 2 growth factors $(24,34)$.

Selected cell types express Ang-1, including megakaryocytes, platelets, and other cells $(35,36)$. Tumors uncommonly express Ang-1 $(37,38)$, but a proportion of bone marrow aspirates from patients with myeloma were found to express Ang-1 (39). When purified from bone marrow, myeloma cells were found to express VEGF-A in most cases and Ang-2 in some cases, but not Ang-1 (40).

Mast cells often represent a dominant infiltrate in human plasma cell malignancies, and the degree of mast cell infiltration parallels the severity of disease $(11,20,21)$. Chronic inflammation induced by i.p. injection of mineral oils plays an active role in the development of peritoneal plasma cell tumors in susceptible mouse strains $(4,41,42)$. We have recently observed (unpublished observations) that mast cells are abundant within the inflammatory infiltrate that accompanies oil-induced granulomas and at the periphery of foci of plasmacytomas that arise in the context of oil-induced granulomas. Intense neovascularization was previously found to characterize the oil granulomas (3). Thus, in addition to promoting the growth of plasmacytoma cell lines inoculated in mice, mast cells may play a role in the development of oil-induced plasmacytomas.

Macrophages and the IL- 6 they secrete play an important pathogenetic role in the development of experimental plasmacytomas induced by mineral oils $(4,43,44)$. Pristaneinduced plasmacytomas are often IL-6-dependent in vivo and in vitro, at least initially, and $\mathrm{BALB} / \mathrm{c} \mathrm{IL-} 6^{-/-}$mice are refractory to plasmacytoma induction by pristane (4). In addition, BALB/c mice overexpressing IL-6 develop a striking lymphadenopathy with accumulation of plasma cells in the medullary cords and, within months, manifest plasma cell tumors at sites of plasma cell accumulation $(4,45)$. However, the contribution of monocytes, macrophages, and IL- 6 to the plasmacytoma tumor-promoting effect of mast cells appears to be limited. Mast cells constitutively secrete low-level IL-6 and monocyte chemoattractant protein-1, and express CD40 ligand, which could promote monocyte/ dendritic cell activation and plasmacytoma expansion by interacting with the CD40 receptor expressed on these cells (46-50). We found that murine monocytes do not express the Ang-1 receptor Tie-2 (results not shown), and that monocytes and macrophages are only rarely visualized in plasmacytoma tissues derived from inoculation of plasmacytoma cells alone or with mast cell coinjection. Since VEGF-A can promote monocyte migration (51), plasmacytoma-derived VEGF-A could explain the low-level monocyte infiltration we detected in tumor tissues $(52,53)$.

Sustained angiogenesis is considered one of the hallmarks of cancer (54). Here, we show that mast cells promote angiogenesis and stimulate the growth of plasmacytoma cells in mice. Together, these observations provide further evidence supporting a causal relationship between inflammation and plasma cell tumor growth. 


\section{Methods}

Reagents and cells. Mouse recombinant IL-3, IL-10, IL-6, and SCF were purchased from PeproTech Inc. Human recombinant Ang-1, which activates murine Tie-2 (30), was purchased from R\&D Systems Inc. RPMI 1640 and FBS were purchased from BioSource International Inc. The mouse plasmacytoma cell lines TEPC2027 and TEPC1165SZ were gifts from B. Mock (National Cancer Institute, Bethesda, Maryland, USA). Culture medium of plasmacytoma cell lines consisted of RPMI 1640 medium containing 10\% FBS, $50 \mu \mathrm{M}$ 2-mercaptoethanol; TEPC1165SZ required IL-6 $(5 \mathrm{ng} / \mathrm{ml})$. Bone marrow mast cells, obtained from C57BL/6 mice (National Cancer Institute), were cultured with $10 \mathrm{ng} / \mathrm{ml} \mathrm{IL-3} \mathrm{(these}$ are referred to here as BMMCs) or with $100 \mathrm{ng} / \mathrm{ml} \mathrm{SCF}$ plus $50 \mathrm{ng} / \mathrm{ml}$ IL-6 plus $10 \mathrm{ng} / \mathrm{ml}$ IL-10 (these are referred to as TRIAD mast cells) as described previously $(55,56)$. SPMCs, derived from spleen of C57BL/6 mice, were cultured with $10 \mathrm{ng} / \mathrm{ml} \mathrm{IL-3.} \mathrm{After} \mathrm{4-5} \mathrm{weeks} \mathrm{of} \mathrm{culture,} \mathrm{more}$ than $98 \%$ of cells were identified as mast cells by toluidine blue staining or flow cytometry for surface c-kit and FceRI. Murine primary monocytes and macrophages were derived from the bone marrow of C57BL/6 mice, as previously described (57). The identity and purity of the monocyte/ macrophage cell population was evaluated by flow cytometry after staining with rat anti-mouse mAb F4/80 (Caltag Laboratories) (58). The murine myelomonocytic cell line NSF-60 (59) was a gift of S. Morse (NIH, Bethesda, Maryland, USA).

RNA preparation and RT-PCR analysis. Total RNA was extracted using TRI Reagent (Molecular Research Center Inc.). cDNA was synthesized from $5 \mu \mathrm{g}$ total RNA using SuperScript preamplification system (Invitrogen Corp.) followed by DNase I treatment (Invitrogen Corp.). Amplification was performed in a $25-\mu 1$ reaction mixture using $0.5 \mu \mathrm{l}$ cDNA, platinum Pf $x$ Taq DNA polymerase (0.25 $\mu \mathrm{l}$; Invitrogen Corp.), $0.5 \mu \mathrm{l}$ dNTP mixture (10 mM; Invitrogen Corp.), and $1 \mu \mathrm{l}$ of sense and antisense primer solution (50 pM). RNA quality was evaluated in all samples by parallel RT-PCR for GAPDH. Absence of contaminating genomic DNA was ensured by RNA-PCR. PCR products were separated on $2 \%$ agarose gel (Invitrogen Corp.) prestained with $1 \mu \mathrm{g} /$ $\mathrm{ml}$ ethidium bromide and visualized under UV light. Primers for VEGF-D, Ang-2, VEGFR1, VEGFR2, and Tie-2 were purchased from R\&D Systems Inc. Other primers include VEGF-A, sense 5'-GCGGGCTGCCTCGCAGTC-3' and antisense 5'-TCACCGCCTTGGCTTGTCA-3'; VEGF-B, sense 5'-CACAGCCAATGTGAATGCA- $3^{\prime}$ and antisense $5^{\prime}$-GCTCTAAGCCCCGCCCTTGGCAATGGAGGAA-3'; VEGF-C, sense 5'-CCAAACCAGTCACAATCAG-3' and antisense $5^{\prime}$-ATTCACAGGCACATTTC-3'; PlGF, sense $5^{\prime}$-TTTCTCAGGATGTGCTCTGTGAA-3' and antisense $5^{\prime}$-CCTGGTTACCTCCGGGAAATGAC-3'; and Ang-1, sense 5'-ATCTACACTATTTATTTTAATAAT-3' and antisense 5'-AAAGTCCAAGGGCCGGATCATCAT-3'.

Assays for determination of VEGF-A, VEGF-C, Ang-1, Ang-2, and Tie-2. VEGF-A content in supernatants of plasmacytoma and mast cells (cultured for 48 hours at $1 \times 10^{6}$ cells $/ \mathrm{ml}$ in complete culture medium) was measured by ELISA (R\&D Systems Inc.). Cell lysates from $2 \times 10^{6}$ mast cells, or plasmacytoma cells, were solubilized in Tricine SDS sample buffer (Novex), boiled, and run through 10\% NuPAGE Novex Bis-Tris Gel (Invitrogen Corp.). After transfer, nitrocellulose membranes (Invitrogen Corp.) were incubated overnight with rabbit anti-VEGF-C antibody (H-190; Santa Cruz Biotechnology Inc.), goat anti-Ang-1 antibody (N-18; Santa Cruz Biotechnology Inc.), goat anti-Ang-2 antibody (F-18; Santa Cruz Biotechnology Inc.), or rabbit anti-Tie-2 antibody (H-176; Santa Cruz Biotechnology Inc.). Bound antibody was detected with affinity-purified, peroxidase-linked, anti-goat IgG Fc antibody or anti-rabbit IgG Fc antibody (Calbiochem; EMD Biosciences Inc.) and a chemiluminescence detection system (ECL kit; Amersham Pharmacia Biotech). Loading accuracy was evaluated by membrane rehybridization with mouse monoclonal anti-actin antibody (Oncogene; EMD Biosciences Inc.).
In vitro functional studies. The effects of Ang-1 on plasmacytoma TEPC1165SZ and TEPC2027 cell proliferation were assessed as described previously (60). Cells were washed twice with PBS, suspended in culture medium (RPMI containing 10\% FBS and $5 \mathrm{ng} / \mathrm{ml} \mathrm{IL-6),}$ plated (TEPC1165SZ, 2,000 cells per well; TEPC2027, 20,000 cells per well in $0.2 \mathrm{ml}$ culture medium) in triplicate with or without $250 \mathrm{ng} / \mathrm{ml}$ Ang-1 onto 96-well plates, and incubated for 64 hours. DNA synthesis was measured by ${ }^{3} \mathrm{H}$-thymidine deoxyribose uptake $(0.5 \mu \mathrm{Ci} /$ well; New England Nuclear; PerkinElmer Inc.) during the last 16 hours of culture. Radioactivity was measured by scintillation counting (Wallac TriLux Betaplate; PerkinElmer Inc.) after cell harvesting onto glass-fiber filtermates (Harvester 96 Mach III; Tomtec). To evaluate the effects of Ang-1 on TEPC1165SZ and TEPC2027 cell survival after serum depletion, cells were washed twice with PBS, suspended in RPMI containing 1\% FBS and $5 \mathrm{ng} / \mathrm{ml} \mathrm{IL}-6$, plated $(100,000$ cells $/ \mathrm{ml})$ in triplicate onto $24-w e l l$ plates with or without $250 \mathrm{ng} / \mathrm{ml}$ Ang-1, and incubated for 48 hours, and viability was determined by trypan blue exclusion.

Immunobistochemistry for Ang-1 and $\kappa$ chain. Immunohistochemical detection of Ang- 1 and $\kappa$ chain was carried out on sections from paraffin-embedded tissues fixed in 10\% neutral-buffered formalin solution (Sigma-Aldrich) using streptavidin-biotinylated HRP detection (Vector Laboratories Inc.). For Ang-1 detection, antigen retrieval was performed by heating of tissue sections in a microwave oven for 10 minutes, and nonspecific binding was prevented by incubation with PBS containing 2\% BSA (PBSA). Tissue sections were incubated with Tie-2/Fc chimera (Sigma-Aldrich) diluted to $5 \mu \mathrm{g} / \mathrm{ml}$ in $2 \%$ PBSA containing $0.6 \%$ Triton X. Human IgG1 Fc (Accurate Chemical \& Scientific Corp.) was used as a control for Tie-2/Fc. 3,3'-diaminobenzidine (DAB; Vector Laboratories Inc.) was used as chromogen, and sections were counterstained with hematoxylin or toluidine blue. $\kappa$ Light chain staining was performed using goat anti- $\kappa$ chain antibody (a gift of E. Mushinski, National Cancer Institute) followed by a donkey anti-goat Ig biotin-conjugated secondary antibody (Sigma-Aldrich).

In vivo Matrigel angiogenesis assay, and measurement of vascular areas and plasmacytoma cell infiltration. The Matrigel angiogenesis assay was performed as described previously (60). Matrigel $(0.5 \mathrm{ml})$, a crude extract of the Engelbreth-Holm-Swarm tumor (BD Biosciences - Discovery Labware) was mixed with plasmacytoma cells $\left(0.5 \times 10^{6}\right.$ or $\left.1.0 \times 10^{6}\right)$, mast cells $\left(0.5 \times 10^{6}\right)$, a mixture of plasmacytoma cells and mast cells $\left(0.5 \times 10^{6}\right.$ of each), or plasmacytoma cells $\left(0.5 \times 10^{6}\right)$ with recombinant Ang-1 (500 $\mathrm{ng} / \mathrm{ml}$ ), and then injected s.c. into the mid-abdominal region of female $\mathrm{BALB} / \mathrm{c} \mathrm{nu} / \mathrm{nu} 6$ - to 8 -week-old mice (3-5 mice per group). All animal experiments were performed under protocols approved by the National Cancer Institute's animal-studies review board. For Ang-1 neutralization in Matrigel, we used $5 \mu \mathrm{g} / \mathrm{ml}$ soluble Tie-2/Fc chimera (61), and B7-1/Fc chimera (R\&D Systems Inc.) was used as control. For VEGF-A neutralization, we used $5 \mu \mathrm{g} / \mathrm{ml}$ goat anti-mouse VEGF-A neutralizing antibody (AF-493-NA; R\&D Systems Inc.), and polyclonal goat IgG (R\&D Systems Inc.) was used as control. After 8 days, Matrigel plugs were removed, fixed in $10 \%$ neutral-buffered formalin solution (Sigma-Aldrich), and embedded in paraffin. Tissues were sectioned ( $5 \mu \mathrm{m}$ thickness), and slides were stained with Masson's trichrome (Histoserv Inc.). Vascular structures were identified as luminal or slitlike structures lined by endothelium, which contained red blood cells. Plasmacytoma cells were identified as cells immunostained for $\kappa$ light chain. Quantitative analysis of angiogenesis and plasmacytoma cell infiltration was performed with the assistance of IPLab software (BioVision Technologies) by scanning of the entire plug section (measuring approximately $0.6 \mathrm{~cm}^{2} ; 10-12$ high-powered fields using a $\times 20$ lens). To measure angiogenesis, we counted the areas occupied by vascular structures; to measure plasmacytoma cell infiltration, we counted the number of plamacytoma cells. We obtained a value from each 
plug section (1 plug section per mouse) and then averaged the results from individual plug sections in each group (3-5 plugs per group). Group values reflect the average readings from all sections in the group. The results of angiogenesis measurements are expressed as the mean area ( \pm SEM; expressed in square micrometers) occupied by vascular structures within a Matrigel field measuring $1.0 \times 10^{6} \mu \mathrm{m}^{2}$; the results of plasmacytoma cell infiltration are expressed as the mean number $( \pm$ SEM) of cells within a Matrigel field measuring $1 \mathrm{~mm}^{2}$.

Plasmacytoma transplantation assays. Four-week-old female BALB/c nu/nu mice (National Cancer Institute), maintained in pathogen-limited conditions, were inoculated s.c. in the flank region above the hind leg with TEPC1165SZ $\left(5 \times 10^{6}\right.$ cells in RPMI); with TEPC2027 $\left(5 \times 10^{6}\right.$ or $10 \times 10^{6}$ cells in RPMI) either alone or mixed with BMMCs $\left(5 \times 10^{6}\right.$ cells in RPMI), SPMCs $\left(5 \times 10^{6}\right.$ cells in RPMI), or freshly separated murine (C57BL/6) bone marrow cells $\left(5 \times 10^{6}\right.$ cells in RPMI); or with BMMCs alone $\left(5 \times 10^{6}\right.$ cells in RPMI) or SPMCs alone $\left(5 \times 10^{6}\right.$ cells in RPMI). The total injection volume was $200 \mu \mathrm{l}$. To study the effects of Ang-1 and VEGF-A neutralization on tumor growth, TEPC1165SZ cells $\left(3 \times 10^{6}\right.$ in RPMI) were inoculated s.c. into 4-week-old female BALB/c nu/nu mice (4 mice per group) either alone or with BMMCs $\left(3 \times 10^{6}\right.$ in RPMI). Together with cell inoculums (TEPC1165SZ alone or TEPC1165SZ plus BMMCs), mice (4 per group) received Tie-2/Fc chimera (50 $\mu$ g per mouse; R\&D Systems Inc.), goat anti-mouse VEGF-A neutralizing antibody ( $50 \mu \mathrm{g}$ per mouse; R\&D Systems Inc.), Tie-2/Fc plus goat anti-mouse VEGF-A antibodies (50 $\mu \mathrm{g}$ each per mouse), or human IgG Fc (50 $\mu$ g per mouse; Accurate Chemical \& Sci- entific Corp.) plus polyclonal goat IgG (50 $\mu \mathrm{g}$ per mouse; R\&D Systems Inc.). The total injection volume was $250 \mu \mathrm{l}(100 \mu \mathrm{l}$ consisted of Matrigel). All animals were examined twice per week; tumor size was estimated in square millimeters as the product of 2-dimensional caliper measurements (longest perpendicular length and width). The percentage tumor size was calculated as the ratio of tumor size with inhibitors or controls (Tie-2/Fc, anti-VEGF-A, IgG/Fc, and control antibody) to tumor size without inhibitors or controls (plasmacytoma cells plus mast cells).

Statistical analysis. Statistical significance of group differences was evaluated by Student's $t$ test using Excel software (Microsoft Corp.).

\section{Acknowledgments}

We thank M. Potter for helpful discussions, L. Sierra for technical help, B.A. Mock and S. Janz for sharing of the plasmacytoma cell lines and for advice, and E. Mushinski and S. Pittaluga for help with immunohistochemistry.

Received for publication May 6, 2004, and accepted in revised form September 7, 2004.

Address correspondence to: Giovanna Tosato, Experimental Transplantation and Immunology Branch, National Cancer Institute, Building 10, Room 12C205, 10 Center Drive 1907, Bethesda, Maryland 20892, USA. Phone: (301) 594-9596; Fax: (301) 5949585; E-mail: Tosatog@mail.nih.gov.
1. Balkwill, F., and Mantovani, A. 2001. Inflammation and cancer: back to Virchow? Lancet. 357:539-545.

2. Coussens, L.M., and Werb, Z. 2002. Inflammation and cancer. Nature. 420:860-867.

3. Anderson, A.O., Wax, J.S., and Potter, M. 1985. Differences in the peritoneal response to pristane in BALB/cAnPt and BALB/cJ mice. Curr. Top. Microbiol. Immunol. 122:242-253.

4. Potter, M. 2003. Neoplastic development in plasma cells. Immunol. Rev. 194:177-195.

5. Lennard-Jones, J.E., Melville, D.M., Morson, B.C., Ritchie, J.K., and Williams, C.B. 1990. Precancer and cancer in extensive ulcerative colitis: findings among 401 patients over 22 years. Gut. 31:800-806.

6. Ernst, P.B., and Pappo, J. 2000. Preventive and therapeutic vaccines against Helicobacter pylori: current status and future challenges. Curr. Pharm. Des. 6:1557-1573.

7. Tsukuma, H., et al. 1993. Risk factors for hepatocellular carcinoma among patients with chronic liver disease. N. Engl. J. Med. 328:1797-1801.

8. Baron, J.A., and Sandler, R.S. 2000. Nonsteroidal anti-inflammatory drugs and cancer prevention. Annu. Rev. Med. 51:511-523.

9. Westphal, J.R. 1891. Über mastzellen. In Farbenalytische Untersuchungen. P. Ehrich, editor. Hirschwald. Berlin, Germany. 17-41.

10. Kessler, D.A., Langer, R.S., Pless, N.A., and Folkman, J. 1976. Mast cells and tumor angiogenesis. Int. J. Cancer. 18:703-709.

11. Ribatti, D., et al. 2001. The role of mast cells in tumour angiogenesis. Br. J. Haematol. 115:514-521.

12. Starkey, J.R., Crowle, P.K., and Taubenberger, S. 1988. Mast-cell-deficient W/Wv mice exhibit a decreased rate of tumor angiogenesis. Int. J. Cancer. 42:48-52.

13. Dethlefsen, S.M., Matsuura, N., and Zetter, B.R. 1994. Mast cell accumulation at sites of murine tumor implantation: implications for angiogenesis and tumor metastasis. Invasion Metastasis. 14:395-408.

14. Coussens, L.M., et al. 1999. Inflammatory mast cells up-regulate angiogenesis during squamous epithelial carcinogenesis. Genes Dev. 13:1382-1397.

15. Coussens, L.M., Tinkle, C.L., Hanahan, D., and
Werb, Z. 2000. MMP-9 supplied by bone marrowderived cells contributes to skin carcinogenesis. Cell. 103:481-490.

16. Egeblad, M., and Werb, Z. 2002. New functions for the matrix metalloproteinases in cancer progression. Nat. Rev. Cancer. 2:161-174.

17. Feoktistov, I., Ryzhov, S., Goldstein, A.E., and Biaggioni, I. 2003. Mast cell-mediated stimulation of angiogenesis: cooperative interaction between A2B and A3 adenosine receptors. Circ. Res. 92:485-492.

18. Shaughnessy, J.D., Jr., and Barlogie, B. 2003. Interpreting the molecular biology and clinical behavior of multiple myeloma in the context of global gene expression profiling. Immunol. Rev. 194:140-163.

19. Vanderkerken, K., Asosingh, K., Croucher, P., and Van Camp, B. 2003. Multiple myeloma biology: lessons from the 5TMM models. Immunol. Rev. 194:196-206.

20. Ribatti, D., et al. 1999. Bone marrow angiogenesis and mast cell density increase simultaneously with progression of human multiple myeloma. Br. J. Cancer. 79:451-455.

21. Vacca, A., Ribatti, D., Roccaro, A.M., Frigeri, A., and Dammacco, F. 2001. Bone marrow angiogenesis in patients with active multiple myeloma. Semin. Oncol. 28:543-550.

22. Ferrara, N., Gerber, H.P., and LeCouter, J. 2003. The biology of VEGF and its receptors. Nat. Med. 9:669-676.

23. Carmeliet, P. 2003. Angiogenesis in health and disease. Nat. Med. 9:653-660.

24. Thurston, G., et al. 1999. Leakage-resistant blood vessels in mice transgenically overexpressing angiopoietin-1. Science. 286:2511-2514.

25. Gurish, M.F., and Austen, K.F. 2001. The diverse roles of mast cells. J. Exp. Med. 194:F1-F5.

26. Norrby, K. 2002. Mast cells and angiogenesis. APMIS. 110:355-371.

27. Davis, S., et al. 1996. Isolation of angiopoietin-1, a ligand for the TIE2 receptor, by secretion-trap expression cloning. Cell. 87:1161-1169.

28. Holash, J., et al. 1999. Vessel cooption, regression, and growth in tumors mediated by angiopoietins and VEGF. Science. 284:1994-1998.
29. Davis, S., et al. 2003. Angiopoietins have distinct modular domains essential for receptor binding, dimerization and superclustering. Nat. Struct. Biol. 10:38-44.

30. Hattori, K., et al. 2001. Vascular endothelial growth factor and angiopoietin-1 stimulate postnatal hematopoiesis by recruitment of vasculogenic and hematopoietic stem cells. J. Exp. Med. 193:1005-1014.

31. Stoeltzing, O., et al. 2003. Angiopoietin-1 inhibits vascular permeability, angiogenesis, and growth of hepatic colon cancer tumors. Cancer Res. 63:3370-3377.

32. Suri, C., et al. 1996. Requisite role of angiopoietin-1, a ligand for the TIE2 receptor, during embryonic angiogenesis. Cell. 87:1171-1180.

33. Sato, T.N., et al. 1995. Distinct roles of the receptor tyrosine kinases Tie- 1 and Tie- 2 in blood vessel formation. Nature. 376:70-74.

34. Thurston, G., et al. 2000. Angiopoietin-1 protects the adult vasculature against plasma leakage. $\mathrm{Nat}$. Med. 6:460-463.

35. Li, J.J., Huang, Y.Q., Basch, R., and Karpatkin, S. 2001. Thrombin induces the release of angiopoietin1 from platelets. Thromb. Haemost. 85:204-206.

36. Metheny-Barlow, L.J., and Li, L.Y. 2003. The enigmatic role of angiopoietin-1 in tumor angiogenesis. Cell Res. 13:309-317.

37. Takahama, M., et al. 1999. Enhanced expression of Tie2, its ligand angiopoietin-1, vascular endothelial growth factor, and CD31 in human non-small cell lung carcinomas. Clin. Cancer Res. 5:2506-2510.

38. Brown, L.F., Dezube, B.J., Tognazzi, K., Dvorak, H.F., and Yancopoulos, G.D. 2000. Expression of Tie1, Tie2, and angiopoietins 1, 2, and 4 in Kaposi's sarcoma and cutaneous angiosarcoma. Am. J. Pathol. 156:2179-2183.

39. Giuliani, N., et al. 2003. Proangiogenic properties of human myeloma cells: production of angiopoietin-1 and its potential relationship to myelomainduced angiogenesis. Blood. 102:638-645.

40. Uneda, S., et al. 2003. Expressions of vascular endothelial growth factor and angiopoietin-2 in myeloma cells. Haematologica. 88:113-115. 
41. Potter, M., and Boyce, C.R. 1962. Induction of plasma-cell neoplasms in strain BALB/c mice with mineral oil and mineral oil adjuvants. Nature. 193:1086-1087.

42. Potter, M., Wax, J.S., Anderson, A.O., and Nordan, R.P. 1985. Inhibition of plasmacytoma development in BALB/c mice by indomethacin. J. Exp. Med. 161:996-1012.

43. Nordan, R.P., and Potter, M. 1986. A macrophagederived factor required by plasmacytomas for survival and proliferation in vitro. Science. 233:566-569.

44. Kishimoto, T. 1989. The biology of interleukin-6. Blood. 74:1-10.

45. Suematsu, S., et al. 1992. Generation of plasmacytomas with the chromosomal translocation $\mathrm{t}(12 ; 15)$ in interleukin 6 transgenic mice. Proc. Natl. Acad. Sci. U. S. A. 89:232-235.

46. Gomi, K., Zhu, F.G., and Marshall, J.S. 2000. Prostaglandin E2 selectively enhances the IgE-mediated production of IL- 6 and granulocyte-macrophage colony-stimulating factor by mast cells through an EP1/EP3-dependent mechanism. J. Immunol. 165:6545-6552.

47. Saitoh, S., et al. 2000. LAT is essential for Fc(epsilon)RI-mediated mast cell activation. Immunity. 12:525-535.

48. Matsushima, K., Larsen, C.G., DuBois, G.C., and Oppenheim,J.J. 1989. Purification and characteriza- tion of a novel monocyte chemotactic and activating factor produced by a human myelomonocytic cell line. J. Exp. Med. 169:1485-1490.

49. Fanslow, W.C., et al. 1994. Structural characteristics of CD40 ligand that determine biological function. Semin. Immunol. 6:267-278.

50. Tournilhac, O., et al. 2004. Excess bone marrow mast cells constitutively express CD154 (CD40 ligand) in Waldenstrom's macroglobulinemia and may support tumor cell growth through CD154/ CD40 pathway [abstract]. J. Clin. Oncol. 22:6555a.

51. Campbell, J.J., et al. 1998. Chemokines and the arrest of lymphocytes rolling under flow conditions. Science. 279:381-384.

52. De Vos, J., et al. 2001. Identifying intercellular signaling genes expressed in malignant plasma cells by using complementary DNA arrays. Blood. 98:771-780.

53. Vande Broek, I., et al. 2003. Chemokine receptor CCR2 is expressed by human multiple myeloma cells and mediates migration to bone marrow stromal cell-produced monocyte chemotactic proteins MCP-1, -2 and -3. Br. J. Cancer. 88:855-862.

54. Hanahan, D., and Weinberg, R.A. 2000. The hallmarks of cancer. Cell. 100:57-70.

55. Masuda, A., Matsuguchi, T., Yamaki, K., Hayakawa, T., and Yoshikai, Y. 2001. Interleukin-15 prevents mouse mast cell apoptosis through STAT6-mediated
Bcl-xL expression. J. Biol. Chem. 276:26107-26113.

56. Yuan, Q.G., Gurish, M.F., Friend, D.S., Austen, K.F., and Boyce, J.A. 1998. Generation of a novel stem cell factor-dependent mast cell progenitor. J. Immunol. 161:5143-5146.

57. Falk, L.A., Wahl, L.M., and Vogel, S.N. 1988. Analysis of Ia antigen expression in macrophages derived from bone marrow cells cultured in granulocytemacrophage colony-stimulating factor or macrophage colony-stimulating factor. J. Immunol. 140:2652-2660.

58. Whiteland, J.L., et al. 1995. Immunohistochemical detection of T-cell subsets and other leukocytes in paraffin-embedded rat and mouse tissues with monoclonal antibodies. J. Histochem. Cytochem. 43:313-320.

59. Pawlak, G., et al. 1999. Colony-stimulating factor-1 impairs both proliferation and differentiation signals of erythropoietin during the commitment of bipotential NFS-60 cell line to the monocytic lineage. Exp. Hematol. 27:797-805.

60. Pike, S.Y., et al. 1998. Vasostatin, a calreticulin fragment, inhibits angiogenesis and suppresses tumor growth. J. Exp. Med. 188:2349-2356.

61. Lin, P., et al. 1997. Inhibition of tumor angiogenesis using a soluble receptor establishes a role for Tie2 in pathologic vascular growth. J. Clin. Invest. 100:2072-2078. 